\title{
Distance-Based Position Analysis of the Three Seven-Link Assur Kinematic Chains
}

\author{
Nicolás Rojas and Federico Thomas \\ Institut de Robòtica i Informàtica Industrial (CSIC-UPC) \\ Llorens Artigues 4-6, 08028 Barcelona, Spain \\ \{nrojas, fthomas\}@iri.upc.edu
}

\begin{abstract}
The position analysis of planar linkages has been dominated by resultant elimination and tangent-half-angle substitution techniques applied to sets of kinematic loop equations. This analysis is thus reduced to finding the roots of a polynomial in one variable, the characteristic polynomial of the linkage. In this paper, by using a new distance-based technique, it is shown that this standard approach becomes unnecessarily involved when applied to the position analysis of the three seven-link Assur kinematic chains. Indeed, it is shown that the characteristic polynomials of these linkages can be straightforwardly derived without relying on variable eliminations nor trigonometric substitutions, and using no others tools than elementary algebra.
\end{abstract}

Keywords: Assur kinematic chains, position analysis, distance-based formulations, Cayley-Menger determinants, bilateration.

\section{Introduction}

A planar linkage is a set of rigid bodies, also called links, pairwise articulated through revolute or slider joints, all lying in a plane. A linkage configuration is an assignment of positions and orientations to all links that respects the kinematic constraints imposed by all joints. The position analysis of a linkage consists in obtaining a complete characterization of its valid configurations.

At the beginning of the twentieth century, the Russian mathematician L.W. Assur proposed a structural classification of planar linkages based on the smallest kinematic chains which, when added to, or subtracted from a linkage, results in a linkage that has the same mobility. Thereafter, these elementary linkages have been called Assur groups. The relevance of these chains become evident when analyzing a complex planar linkage, because it is always possible to decompose it into Assur groups which can be analyzed one-by-one. A linkage, with no mobility, from which an Assur kinematic group is obtained by removing any one of its links is defined as an Assur kinematic chain (AKC) or Baranov truss when no slider joints are considered. Hence an AKC corresponds to multiple Assur groups.

Considering only revolute pairs - a prismatic pair can be modeled as a limit case of a revolute pair-, the simplest $\mathrm{AKC}$ is the well-known triad, a one-loop structure with 


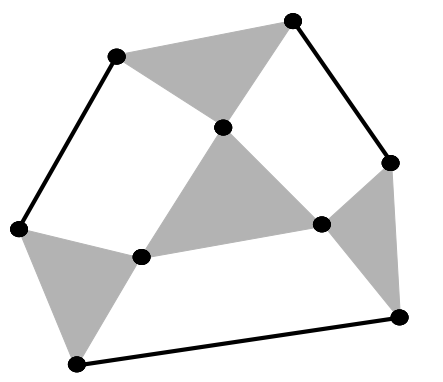

Type I

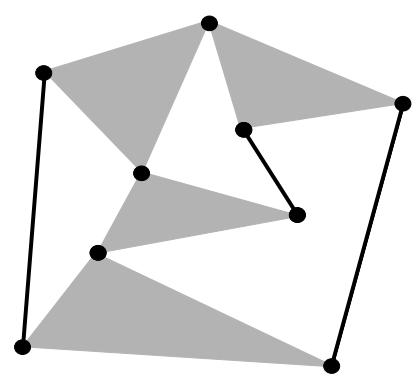

Type II

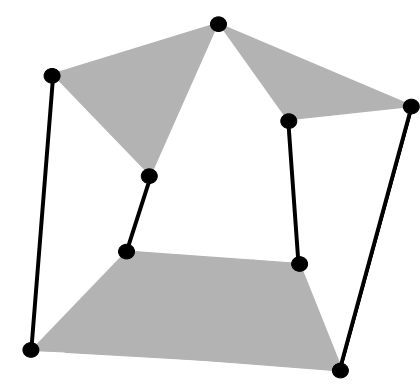

Type III

Figure 1: The three seven-link Assur kinematic chains.

three links and two assembly modes. There is one AKC with two loops, the pentad, a five-link structure whose position analysis leads to up to 6 assembly modes. E. Peysah is credited to be the first researcher in obtaining an analytic form solution for this problem in 1985 1], the same result was obtained independently at least by G. Pennock and D. Kassner [2], K. Wohlhart [3], and C. Gosselin et al. [4]. More recently, N. Rojas and F. Thomas [5] showed that this result can be obtained, in a straightforward way, using bilaterations. Regarding three loops, or seven links, there are three types of AKCs (see Fig. 1), namely, I) a linkage with three binary links and four ternary links with one ternary link connected to the other three, II) a linkage with three binary links and four serially-connected ternary links, and III) a linkage with four binary links, two ternary links, and one quaternary link. The position analysis of these linkages leads to up to 14, 16, and 18 assembly modes, respectively. C. Innocenti, in [6], 7] and [8], obtained these results using resultant elimination techniques. Alternatively, for the type I seven-link AKC, a solution based on homotopy continuation was presented by A. Liu and T. Yang in [9]. We show herein, by extending the ideas presented in [5], that a formulation based on bilaterations leads to the same results presented by C. Innocenti in his three articles in a more straightforward way.

The position analysis of planar linkages has been dominated by resultant elimination and tangent-half-angle substitution techniques applied to sets of kinematic loop equations. This analysis is thus reduced to finding the roots of a polynomial in one variable, the characteristic polynomial of the linkage. A. Dhingra and col. used reduced Gröbner bases and Sylvester's elimination to obtain these polynomials [10]. J. Nielsen and B. Roth also gave an elimination-based method that uses Dixon's resultant to derive the lowest degree characteristic polynomials [11]. This technique was later improved by C. Wampler [12], who used a complex-plane formulation to reduce the size of the final eigenvalue problem by half. The position analysis of planar linkages has also been tackled using general continuation-based solvers [13, 14], that start with a system whose solutions are known, and then transform it gradually into the system whose solutions are sought, while tracking all solution paths along the way [15]. Interval-based methods has also been successfully applied to solve the equations resulting from position analysis problems [16, 17]. Herein, we propose an alternative approach based on bilaterations. The use of bilaterations reduces the number of equations to the point in which no variable elimination is required for the position analysis of the three seven-link Assur kinematic 
chains. Moreover, since a bilateration operation is entirely posed and solved in terms of distances, no tangent-half-angle substitutions are needed.

The rest of the paper is organized as follows. A coordinate-free formula for bilateration expressed in terms of Cayley-Menger determinants is presented in Section 2. In Section 3. it is shown how the distance ratio between any two couples of vertices in a tree of triangles can be obtained by a set of bilaterations. This result is used in Sections 4, 5 , and 6 to derive a distance-based characteristic polynomial for the three seven-Link Assur kinematic chains of type I, II, and III, respectively. Finally, Section 7 summarizes the main points and gives prospects for further research.

\section{Cayley-Menger determinants and bilateration}

Let $P_{i}$ and $\mathbf{p}_{i}$ denote a point and its position vector in a given reference frame, respectively. Then, let us define

$$
D\left(i_{1}, \ldots, i_{n} ; j_{1}, \ldots, j_{n}\right)=2\left(\frac{-1}{2}\right)^{n}\left|\begin{array}{cccc}
0 & 1 & \ldots & 1 \\
1 & s_{i_{1}, j_{1}} & \ldots & s_{i_{1}, j_{n}} \\
\vdots & \vdots & \ddots & \vdots \\
1 & s_{i_{n}, j_{1}} & \ldots & s_{i_{n}, j_{n}}
\end{array}\right|
$$

with $s_{i, j}=d_{i, j}^{2}=\left\|\mathbf{p}_{i j}\right\|^{2}$, where $\mathbf{p}_{i j}=\mathbf{p}_{j}-\mathbf{p}_{i}=\overrightarrow{P_{i} P_{j}}$. This determinant is known as the Cayley-Menger bi-determinant of the point sequences $P_{i_{1}}, \ldots, P_{i_{n}}$, and $P_{j_{1}}, \ldots, P_{j_{n}}$ and its geometric interpretation plays a fundamental role in distance geometry, the analytical study of Euclidean geometry in terms of invariants. When the two point sequences are the same, it is convenient to abbreviate $D\left(i_{1}, \ldots, i_{n} ; i_{1}, \ldots, i_{n}\right)$ by $D\left(i_{1}, \ldots, i_{n}\right)$, which is simply called the Cayley-Menger determinant of the involved points.

The evaluation of $D\left(i_{1}, \ldots, i_{n}\right)$ gives $(n-1) !^{2}$ times the squared hypervolume of the simplex spanned by the points $P_{i_{1}}, \ldots, P_{i_{n}}$ in $\mathbb{R}^{n-1}$. Therefore, the squared distance between $P_{i}$ and $P_{j}$ can be expressed as $D(i, j)$ and the signed area 11 of the triangle $P_{i} P_{j} P_{k}$ as $\pm \frac{1}{2} \sqrt{D(i, j, k)}$. It can also be verified that $D\left(i_{1}, i_{2} ; j_{1}, j_{2}\right)$ is equivalent to the dot product between the vectors $\left(\mathbf{p}_{i_{2}}-\mathbf{p}_{i_{1}}\right)$ and $\left(\mathbf{p}_{j_{2}}-\mathbf{p}_{j_{1}}\right)$. Then, $\cos (\theta)=$ $D(i, j ; i, k) / \sqrt{D(i, j) D(i, k)}, \theta$ being $\angle P_{j} P_{i} P_{k}$. For a brief review of the properties of Cayley-Menger determinants, see [18] and the references therein.

Many geometric problems can be elegantly formulated using Cayley-Menger determinants. The bilateration problem is one of them. It consists of finding the feasible locations of a point, say $P_{k}$, given its distances to two other points, say $P_{i}$ and $P_{j}$, whose locations are known. Then, according to Fig. 2 the position vector of the orthogonal projection of $P_{k}$ onto the line defined by $P_{i} P_{j}$ can be expressed as

$$
\mathbf{p}=\mathbf{p}_{i}+\sqrt{\frac{D(i, k)}{D(i, j)}} \cos \theta\left(\mathbf{p}_{j}-\mathbf{p}_{i}\right)=\mathbf{p}_{i}+\frac{D(i, j ; i, k)}{D(i, j)}\left(\mathbf{p}_{j}-\mathbf{p}_{i}\right)
$$

\footnotetext{
${ }^{1}$ For a triangle $P_{i} P_{j} P_{k}$ in the Euclidean plane with area $A$, the signed area is defined as $+A$ (respectively, $-A$ ) if the point $P_{j}$ is to the right (respectively to the left) of the line $P_{i} P_{k}$, when going from $P_{i}$ to $P_{k}$
} 


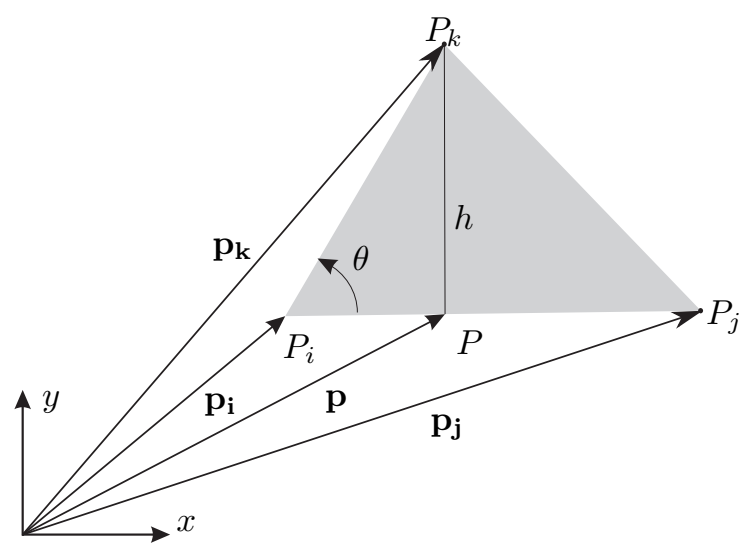

Figure 2: The bilateration problem in $\mathbb{R}^{2}$.

Moreover, the position vector of $P_{k}$ can be expressed as

$$
\mathbf{p}_{k}=\mathbf{p} \pm \frac{\sqrt{D(i, j, k)}}{D(i, j)} \mathbf{S}\left(\mathbf{p}_{j}-\mathbf{p}_{i}\right)
$$

where $\mathbf{S}=\left(\begin{array}{cc}0 & -1 \\ 1 & 0\end{array}\right)$ and the \pm sign accounts for the two mirror symmetric locations of $P_{k}$ with respect to the lines supporting the segment defined by $P_{i} P_{j}$. Then, substituting (2) in (3) and expressing the result in matrix form, we obtain

$$
\mathbf{p}_{i k}=\mathbf{Z}_{i, j, k} \mathbf{p}_{i j}
$$

where

$$
\mathbf{Z}_{i, j, k}=\frac{1}{D(i, j)}\left[\begin{array}{cc}
D(i, j ; i, k) & \mp \sqrt{D(i, j, k)} \\
\pm \sqrt{D(i, j, k)} & D(i, j ; i, k)
\end{array}\right],
$$

which will be called bilateration matrix. Now, it is important to observe that this kind of matrices constitute an Abelian group under product and addition and if $\mathbf{v}=\mathbf{Z} \mathbf{w}$, where $\mathbf{Z}$ is a bilateration matrix, then $\|\mathbf{v}\|^{2}=\operatorname{det}(\mathbf{Z})\|\mathbf{w}\|^{2}$.

In what follows, in order to simplify the notation, we will abbreviate the product $\mathbf{Z}_{i, j, k} \mathbf{Z}_{i, k, l}$ by $\boldsymbol{\Omega}_{i, j, k, l}$.

\section{Distance ratios in trees of triangles}

A tree of triangles is defined as a set of triangles that are connected by their edges such that any two triangles are connected by a single strip of triangles, i.e. a series of connected triangles that share one edge with one neighbor and another with the next [Fig. 3(left)]. Note that this definition includes cases in which edges are shared by more than two triangles.

In a tree of triangles, it is straightforward to find the ratio between any two distances involving any couple of vertices using sequences of bilaterations. This is better explained 

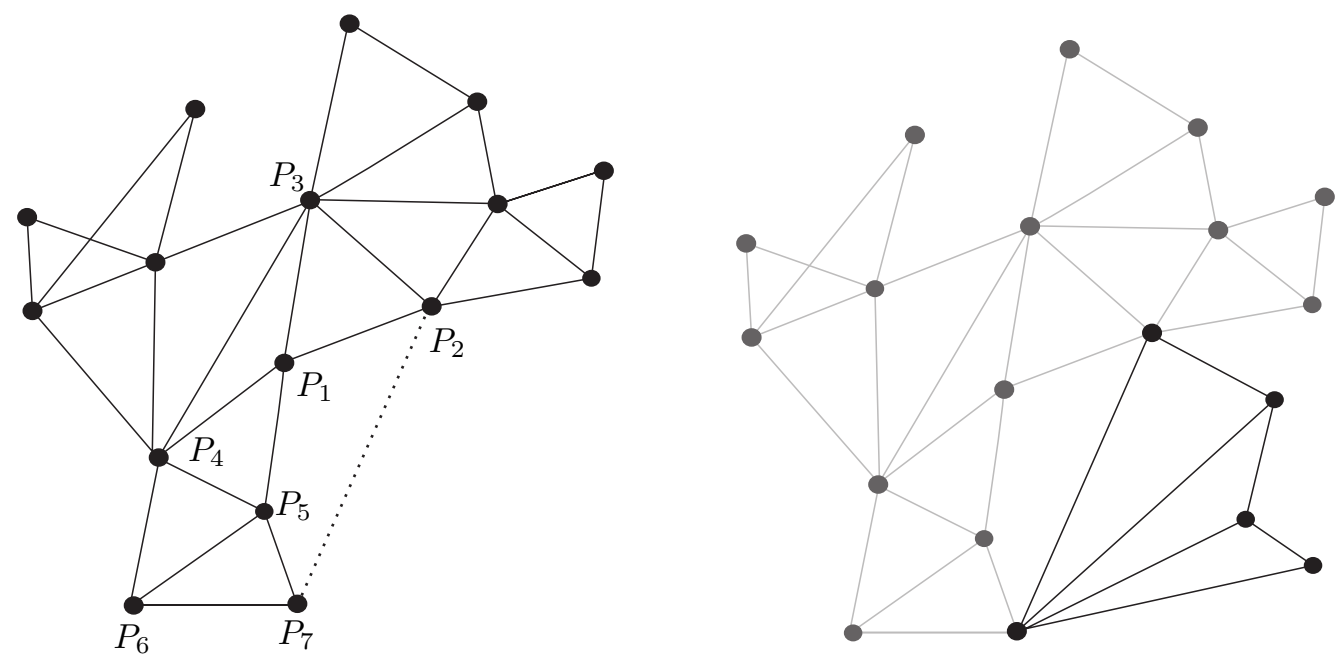

Figure 3: Left: In a tree of triangles the distance ratio between two couples of vertices can be obtained by a set of bilaterations (see text for details). Right: The same is possible for arbitrary couples of vertices belonging to two trees of triangles sharing two vertices.

through an example. Let us suppose that we are interested in finding $\frac{s_{5,7}}{s_{1,2}}$ in the tree of triangles in Fig. 3(left). The corresponding edges are connected by the strip of triangles $\left\{P_{1} P_{2} P_{3}, P_{1} P_{3} P_{4}, P_{1} P_{4} P_{5}, P_{5} P_{4} P_{6}, P_{5} P_{6} P_{7}\right\}$. Then, taking the segment $P_{1} P_{2}$ as reference, we can perform the following sequence of bilaterations:

$$
\begin{aligned}
\mathbf{p}_{1,3} & =\mathbf{Z}_{1,2,3} \mathbf{p}_{1,2} \\
\mathbf{p}_{1,4} & =\mathbf{Z}_{1,3,4} \mathbf{p}_{1,3}=\mathbf{Z}_{1,3,4} \mathbf{Z}_{1,2,3} \mathbf{p}_{1,2} \\
\mathbf{p}_{1,5} & =\mathbf{Z}_{1,4,5} \mathbf{p}_{1,4}=\mathbf{Z}_{1,4,5} \mathbf{Z}_{1,3,4} \mathbf{Z}_{1,2,3} \mathbf{p}_{1,2} \\
\mathbf{p}_{5,6} & =\mathbf{Z}_{5,4,6} \mathbf{p}_{5,4}=\mathbf{Z}_{5,4,6}\left(\mathbf{p}_{1,4}-\mathbf{p}_{1,5}\right) \\
& =\mathbf{Z}_{5,4,6}\left(\mathbf{I}-\mathbf{Z}_{1,4,5}\right) \mathbf{Z}_{1,3,4} \mathbf{Z}_{1,2,3} \mathbf{p}_{1,2} \\
\mathbf{p}_{5,7} & =\mathbf{Z}_{5,6,7} \mathbf{p}_{5,6}=\mathbf{Z}_{5,6,7} \mathbf{Z}_{5,4,6}\left(\mathbf{I}-\mathbf{Z}_{1,4,5}\right) \mathbf{Z}_{1,3,4} \mathbf{Z}_{1,2,3} \mathbf{p}_{1,2} .
\end{aligned}
$$

As a consequence,

$$
\frac{s_{5,7}}{s_{1,2}}=\operatorname{det}\left(\mathbf{Z}_{5,6,7} \mathbf{Z}_{5,4,6}\left(\mathbf{I}-\mathbf{Z}_{1,4,5}\right) \mathbf{Z}_{1,3,4} \mathbf{Z}_{1,2,3}\right)
$$

Now, let us suppose that we want to compute $\frac{s_{2,7}}{s_{1,2}}$. In this case $P_{2} P_{7}$ is not an edge of any triangle but clearly

$$
\mathbf{p}_{2,7}=-\mathbf{p}_{1,2}+\mathbf{p}_{1,5}+\mathbf{p}_{5,7} .
$$

Then, the substitution of (7) and (10) in the above equation yields:

$$
\mathbf{p}_{2,7}=\left(-\mathbf{I}+\left(\mathbf{Z}_{1,4,5}+\mathbf{Z}_{5,6,7} \mathbf{Z}_{5,4,6}\left(\mathbf{I}-\mathbf{Z}_{1,4,5}\right)\right) \mathbf{Z}_{1,3,4} \mathbf{Z}_{1,2,3}\right) \mathbf{p}_{1,2} .
$$


Therefore,

$$
\frac{s_{2,7}}{s_{1,2}}=\operatorname{det}\left(-\mathbf{I}+\left(\mathbf{Z}_{1,4,5}+\mathbf{Z}_{5,6,7} \mathbf{Z}_{5,4,6}\left(\mathbf{I}-\mathbf{Z}_{1,4,5}\right)\right) \mathbf{Z}_{1,3,4} \mathbf{Z}_{1,2,3}\right) .
$$

By proceeding in a similar way, it is possible to obtain the distance ratio between any two couples of points.

The possibility of computing distance ratios that involve arbitrary couples of vertices, using sequences of bilaterations, is not limited to trees of triangles. Observe how this can also be applied to two triangular trees sharing any two vertices [see Fig. 3(right)]. This is the case of the three seven-Link Assur kinematic chains of type I, II, and III, as shown in the next three sections.

\section{Position analysis of the type I seven-link AKC}

Fig. 4 shows the general seven-link AKC of type I. If the central ternary link is assumed to be connected to the ground, the centers of its revolute pairs define the base triangle $P_{3} P_{5} P_{4}$, the revolute pair centers of the other three ternary links define the moving triangles $P_{5} P_{8} P_{9}, P_{4} P_{7} P_{2}$, and $P_{3} P_{1} P_{6}$. The position analysis problem for this linkage consists in, given the dimensions of every link and the position of the centers $P_{3}, P_{4}$, and $P_{5}$, calculating the Cartesian pose of the moving ternary links. Next, a coordinate-free formula for the position analysis of this linkage, without using trigonometrical functions nor resultant methods, is derived. To this end, instead of directly computing the Cartesian pose of the moving ternary links, we will compute the set of values of $s_{2,3}$ compatible with the binary and ternary links side lengths. Thus, this procedure is entirely posed in terms of distances. Actually, we will show how this boils down to compute the distance ratio $\frac{s_{6,8}}{s_{2,3}}$ which can be obtained by considering the two threes of triangles defined by $\left\{P_{1} P_{3} P_{6}, P_{1} P_{2} P_{3}, P_{2} P_{4} P_{3}, P_{2} P_{7} P_{4}, P_{3} P_{4} P_{5}\right\}$ and $\left\{P_{5} P_{7} P_{9}, P_{5} P_{9} P_{8}\right\}$.

\subsection{Scalar equation derivation}

According to Fig. 4, we have

$$
\begin{aligned}
& \mathbf{p}_{2,7}=\mathbf{Z}_{2,4,7} \mathbf{p}_{2,4}=-\mathbf{Z}_{2,4,7} \mathbf{Z}_{2,3,4} \mathbf{p}_{3,2}=-\boldsymbol{\Omega}_{2,3,4,7} \mathbf{p}_{3,2} \\
& \mathbf{p}_{3,5}=\mathbf{Z}_{3,4,5} \mathbf{p}_{3,4}=\mathbf{Z}_{3,4,5} \mathbf{Z}_{3,2,4} \mathbf{p}_{3,2}=\boldsymbol{\Omega}_{3,2,4,5} \mathbf{p}_{3,2} \\
& \mathbf{p}_{3,6}=\mathbf{Z}_{3,1,6} \mathbf{p}_{3,1}=\mathbf{Z}_{3,1,6} \mathbf{Z}_{3,2,1} \mathbf{p}_{3,2}=\boldsymbol{\Omega}_{3,2,1,6} \mathbf{p}_{3,2} \\
& \mathbf{p}_{5,8}=\mathbf{Z}_{5,9,8} \mathbf{p}_{5,9}=\mathbf{Z}_{5,9,8} \mathbf{Z}_{5,7,9} \mathbf{p}_{5,7}=\boldsymbol{\Omega}_{5,7,9,8} \mathbf{p}_{5,7}
\end{aligned}
$$

Since

$$
\mathbf{p}_{6,8}=-\mathbf{p}_{3,6}+\mathbf{p}_{3,5}+\mathbf{p}_{5,8}
$$

and

$$
\mathbf{p}_{5,7}=-\mathbf{p}_{3,5}+\mathbf{p}_{3,2}+\mathbf{p}_{2,7},
$$

then

$$
\begin{aligned}
\mathbf{p}_{6,8} & =-\boldsymbol{\Omega}_{3,2,1,6} \mathbf{p}_{3,2}+\boldsymbol{\Omega}_{3,2,4,5} \mathbf{p}_{3,2}+\boldsymbol{\Omega}_{5,7,9,8} \mathbf{p}_{5,7} \\
& =\left[\begin{array}{ll}
-\boldsymbol{\Omega}_{3,2,1,6}+\boldsymbol{\Omega}_{3,2,4,5}-\boldsymbol{\Omega}_{5,7,9,8}\left(\boldsymbol{\Omega}_{3,2,4,5}+\boldsymbol{\Omega}_{2,3,4,7}-\mathbf{I}\right)
\end{array}\right] \mathbf{p}_{3,2} .
\end{aligned}
$$




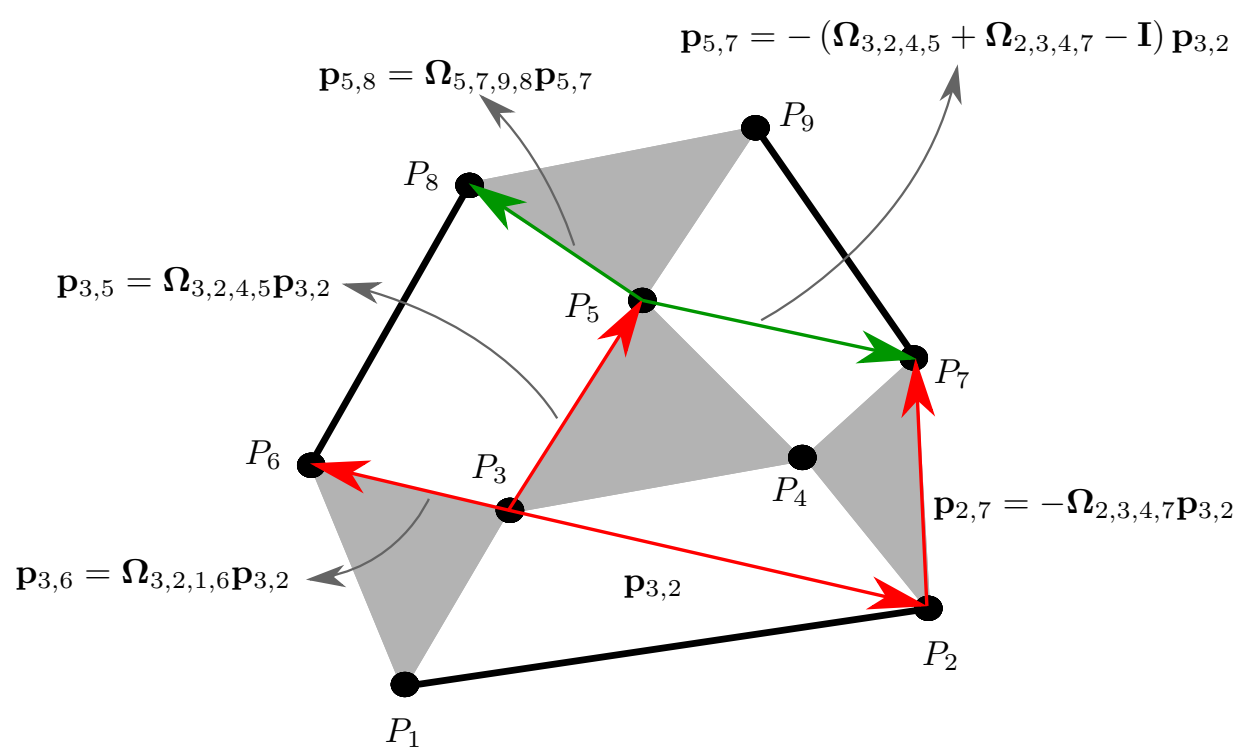

Figure 4: The general seven-link AKC of type I. $\mathbf{p}_{6,8}$ can be expressed in function of $\mathbf{p}_{3,2}$ by computing eight bilaterations.

Therefore,

$$
\operatorname{det}\left(-\boldsymbol{\Omega}_{3,2,1,6}+\boldsymbol{\Omega}_{3,2,4,5}-\boldsymbol{\Omega}_{5,7,9,8}\left(\boldsymbol{\Omega}_{3,2,4,5}+\boldsymbol{\Omega}_{2,3,4,7}-\mathbf{I}\right)\right)=\frac{s_{6,8}}{s_{2,3}} .
$$

The left hand side of the above equation is a function of the unknown squared distances $s_{2,3}$ and $s_{5,7}$. Since, from equation (16),

$$
s_{5,7}=\operatorname{det}\left(\boldsymbol{\Omega}_{3,2,4,5}+\boldsymbol{\Omega}_{2,3,4,7}-\mathbf{I}\right) s_{2,3},
$$

then the substitution of (18) in (17) yields a scalar equation in a single variable: $s_{2,3}$. The roots of this equation, in the range in which the signed areas of the triangles $P_{1} P_{3} P_{2}$ and $P_{3} P_{2} P_{4}$ are real, that is, the range

$$
\left[\max \left\{\left(d_{1,2}-d_{1,3}\right)^{2},\left(d_{2,4}-d_{3,4}\right)^{2}\right\}, \min \left\{\left(d_{1,2}+d_{1,3}\right)^{2},\left(d_{2,4}+d_{3,4}\right)^{2}\right\}\right]
$$

determine the assembly modes of the seven-link AKC of type I. These roots can be readily obtained for the four possible combinations of signs for the signed areas of the triangles $P_{1} P_{3} P_{2}$ and $P_{3} P_{2} P_{4}$ using, for example, an interval Newton method. For each of these roots, we can determine the Cartesian position of the six revolute pair centers of the moving ternary links using equations (11)-(14) and the equation $\mathbf{p}_{2,3}=\mathbf{Z}_{3,4,2} \mathbf{p}_{3,4}$. This process leads up to eight combinations of locations for $P_{6}$ and $P_{8}$, and at least one of them must satisfy the distance imposed by the binary link connecting them.

If a polynomial representation is preferred, despite the previous derivation completely solves the position analysis problem, we can proceed as described next. 


\subsection{Polynomial derivation}

By expanding all the Cayley-Menger determinants involved in equation (18), we get

$$
s_{5,7}=\Gamma_{1}+\Gamma_{2} A_{3,2,4}
$$

where

$$
A_{3,2,4}= \pm \frac{1}{2} \sqrt{\left[s_{2,3}-\left(d_{2,4}-d_{3,4}\right)^{2}\right]\left[\left(d_{2,4}+d_{3,4}\right)^{2}-s_{2,3}\right]},
$$

and $\Gamma_{1}, \Gamma_{2}$ are polynomials in $s_{2,3}$ whose coefficients are algebraic functions of the known squared distances $s_{3,4}, s_{3,5}, s_{4,5}, s_{4,7}, s_{2,7}$, and $s_{2,4}$. On the other hand, by expanding equation (17), we obtain

$$
\begin{aligned}
& \Upsilon_{1}+\Upsilon_{2} A_{3,2,1}+\Upsilon_{3} A_{3,2,4}+\Upsilon_{4} A_{5,7,9}+\Upsilon_{5} A_{3,2,1} A_{3,2,4} \\
& +\Upsilon_{6} A_{3,2,1} A_{5,7,9}+\Upsilon_{7} A_{3,2,4} A_{5,7,9}+\Upsilon_{8} A_{3,2,1} A_{3,2,4} A_{5,7,9}-s_{6,8} s_{2,3} s_{5,7}=0
\end{aligned}
$$

where

$$
\begin{aligned}
& A_{3,2,1}= \pm \frac{1}{2} \sqrt{\left[s_{2,3}-\left(d_{1,2}-d_{1,3}\right)^{2}\right]\left[\left(d_{1,2}+d_{1,3}\right)^{2}-s_{2,3}\right]}, \\
& A_{5,7,9}= \pm \frac{1}{2} \sqrt{\left[s_{5,7}-\left(d_{5,9}-d_{7,9}\right)^{2}\right]\left[\left(d_{5,9}+d_{7,9}\right)^{2}-s_{5,7}\right]}
\end{aligned}
$$

and $\Upsilon_{i}, i=1, \ldots, 8$, are polynomials in $s_{2,3}$ and $s_{5,7}$ whose coefficients are algebraic functions of known distances.

Now, by properly squaring equation (20), we obtain a polynomial equation in $s_{5,7}$ whose coefficients are radical expressions in $s_{2,3}$. Therefore, by replacing (19) into this polynomial equation, we get

$$
\Phi_{1}+\Phi_{2} A_{3,2,1}+\Phi_{3} A_{3,2,4}+\Phi_{4} A_{3,2,1} A_{3,2,4}=0
$$

where $\Phi_{1}, \Phi_{2}, \Phi_{3}$ and $\Phi_{4}$ are polynomials in $s_{2,3}$ of degree $6,5,5$, and 4 , respectively. Finally, the square roots in (21) can be eliminated by properly twice squaring it. This operation yields

$$
\begin{aligned}
& -\Phi_{4}^{4} A_{3,2,1}^{4} A_{3,2,4}^{4}+2 \Phi_{4}^{2} \Phi_{2}^{2} A_{3,2,1}^{4} A_{3,2,4}^{2}+2 \Phi_{4}^{2} \Phi_{3}^{2} A_{3,2,1}^{2} A_{3,2,4}^{4}-\Phi_{2}^{4} A_{3,2,1}^{4} \\
& +\left(2 \Phi_{2}^{2} \Phi_{3}^{2}-8 \Phi_{2} \Phi_{3} \Phi_{4} \Phi_{1}+2 \Phi_{4}^{2} \Phi_{1}^{2}\right) A_{3,2,1}^{2} A_{3,2,4}^{2}-\Phi_{3}^{4} A_{3,2,4}^{4} \\
& +2 \Phi_{1}^{2} \Phi_{2}^{2} A_{3,2,1}^{2}+2 \Phi_{1}^{2} \Phi_{3}^{2} A_{3,2,4}^{2}-\Phi_{1}^{4}=0
\end{aligned}
$$

which, when fully expanded, leads to

$$
s_{2,3}^{4} s_{5,7}^{3} \Delta_{I}=0
$$

where $\Delta_{I}$ is a 14 th-degree polynomial in $s_{2,3}$. The extraneous roots at $s_{2,3}=0$ and $s_{5,7}=0$ were introduced when clearing denominators to obtain equation (20), so they can be dropped. The degree of polynomial $\Delta_{I}$ concurs with the result presented by C. Innocenti in [6]. 

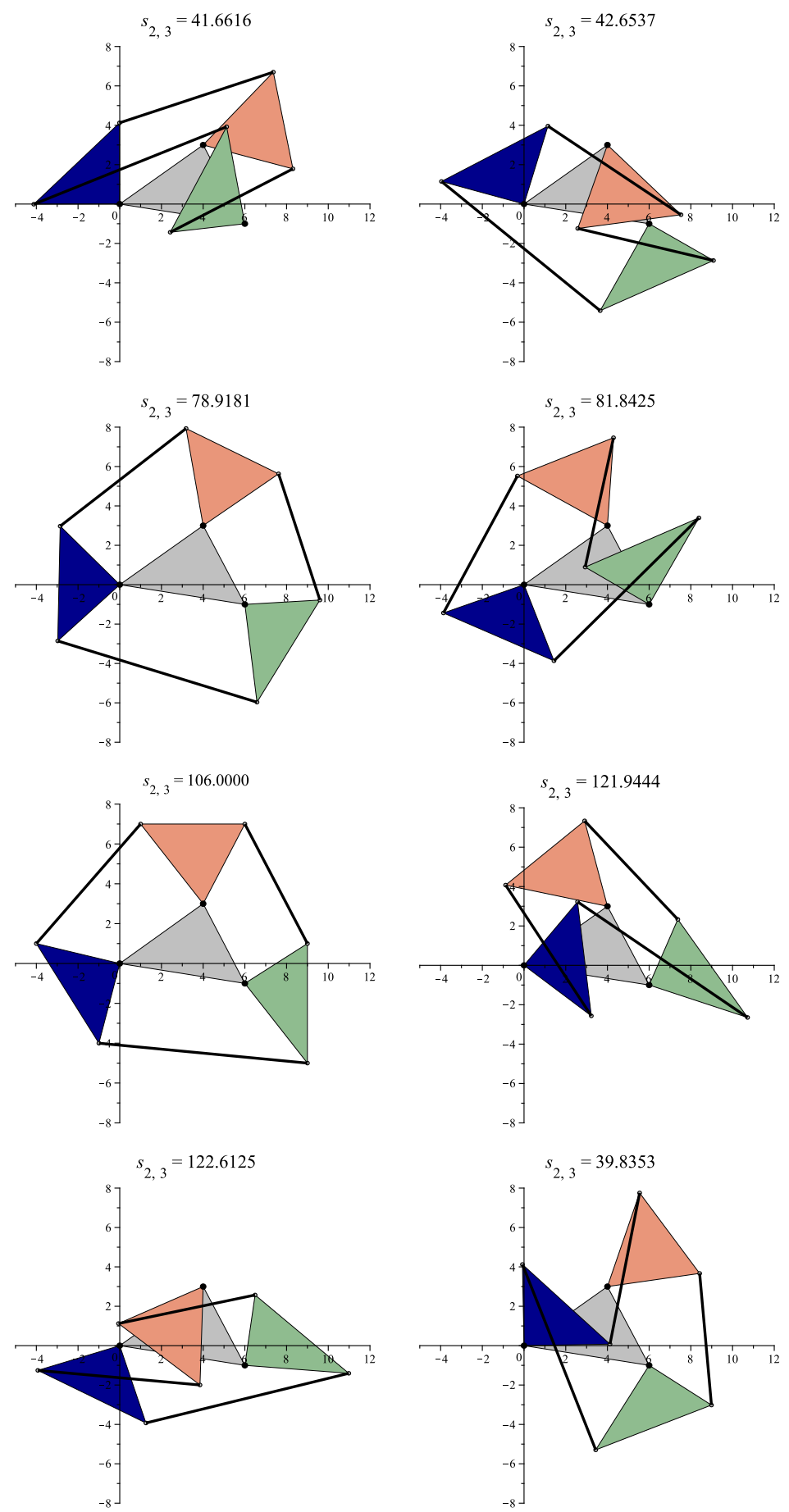

Figure 5: The assembly modes of the analyzed type I seven-link AKC. 


\subsection{Example}

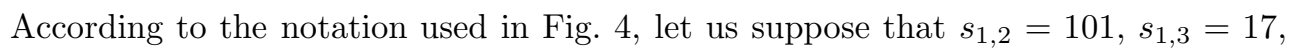
$s_{1,6}=34, s_{2,4}=25, s_{2,7}=36, s_{3,4}=37, s_{3,5}=25, s_{3,6}=17, s_{4,5}=20, s_{4,7}=13$, $s_{5,8}=25, s_{5,9}=20, s_{6,8}=61, s_{7,9}=45$, and $s_{8,9}=25$. Substituting these values in (21), we obtain

$$
\Phi_{1}+\Phi_{2} A_{3,2,1}+\Phi_{3} A_{3,2,4}+\Phi_{4} A_{3,2,1} A_{3,2,4}=0
$$

where

$$
\begin{aligned}
\Phi_{1} & =3.637810^{5} s_{2,3}^{6}-8.279810^{7} s_{2,3}^{5}-6.218910^{8} s_{2,3}^{4}+1.459110^{12} s_{2,3}^{3} \\
& -1.222710^{14} s_{2,3}^{2}+2.881110^{15} s_{2,3}-5.541510^{15} \\
\Phi_{2} & =-9.985010^{5} s_{2,3}^{5}+3.151910^{8} s_{2,3}^{4}-3.283410^{10} s_{2,3}^{3}+1.252810^{12} s_{2,3}^{2} \\
& -1.543010^{13} s_{2,3}+6.024810^{13} \\
\Phi_{3} & =9.985010^{5} s_{2,3}^{5}-4.138310^{8} s_{2,3}^{4}+6.111610^{10} s_{2,3}^{3}-3.713410^{12} s_{2,3}^{2} \\
& +6.651610^{13} s_{2,3}+4.217410^{14} \\
\Phi_{4} & =1.455110^{6} s_{2,3}^{4}-3.585210^{8} s_{2,3}^{3}+3.406410^{10} s_{2,3}^{2}-1.597210^{12} s_{2,3} \\
& +2.199010^{13} \\
A_{3,2,1} & = \pm \frac{1}{2} \sqrt{\left(s_{2,3}-200.8734\right)\left(35.1266-s_{2,3}\right)} \\
A_{3,2,4} & = \pm \frac{1}{2} \sqrt{\left(s_{2,3}-122.8276\right)\left(1.1724-s_{2,3}\right)}
\end{aligned}
$$

Equation (24) is a scalar equation in $s_{2,3}$ which can be numerically solved for the four possible combinations of signs of the two involved squared roots. Alternatively, substituting the above values in $\Delta_{I}$, the following characteristic polynomial is obtained:

$$
\begin{aligned}
& 119.550310^{12} s_{2,3}^{14}-132.808110^{15} s_{2,3}^{13}+67.750710^{18} s_{2,3}^{12}-20.972910^{21} s_{2,3}^{11} \\
& +4.387510^{24} s_{2,3}^{10}-654.047210^{24} s_{2,3}^{9}+71.415110^{27} s_{2,3}^{8}-5.783010^{30} s_{2,3}^{7} \\
& +347.794110^{30} s_{2,3}^{6}-15.405010^{33} s_{2,3}^{5}+492.893010^{33} s_{2,3}^{4}-11.005110^{36} s_{2,3}^{3} \\
& +161.470910^{36} s_{2,3}^{2}-1.388410^{39} s_{2,3}+5.264110^{39} .
\end{aligned}
$$

The real roots of this polynomial are $39.8353,41.6616,42.6537,78.9181,81.8425$, $106.0000,121.9444$, and 122.6125 . The corresponding configurations for the case in which $\mathbf{p}_{3}=(0,0)^{T}, \mathbf{p}_{4}=(6,-1)^{T}$, and $\mathbf{p}_{5}=(4,3)^{T}$ appear in Fig. 5 .

\section{Position analysis of the type II seven-link AKC}

Fig. 6 shows the general seven-link AKC of type II. If the central ternary link is assumed to be connected to the ground, the centers of its revolute pairs define the base triangle $P_{2} P_{4} P_{5}$, the revolute pair centers of the other three ternary links define the moving triangles $P_{4} P_{6} P_{7}, P_{7} P_{9} P_{8}$, and $P_{2} P_{3} P_{1}$. The position analysis problem for this linkage consists in, given the dimensions of every link and the position of the centers $P_{2}$, $P_{4}$, and $P_{5}$, calculating the Cartesian pose of the moving ternary links. Next, following the same strategy as the one used in the previous section, a polynomial in $s_{4,8}$ is derived. 


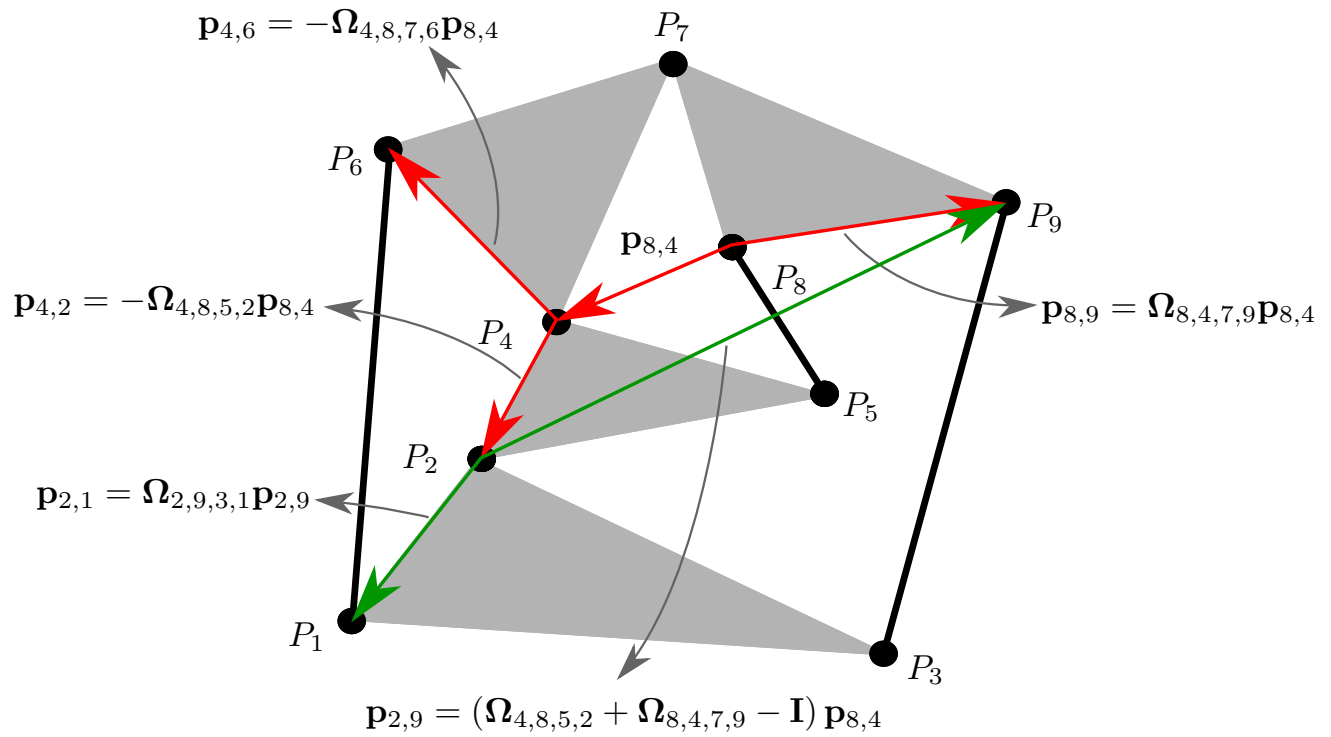

Figure 6: The general seven-link AKC of type II. $\mathbf{p}_{1,6}$ can be expressed in function of $\mathbf{p}_{8,4}$ by computing eight bilaterations.

\subsection{Scalar equation derivation}

According to Fig. 6, we have

$$
\begin{aligned}
& \mathbf{p}_{4,2}=\mathbf{Z}_{4,5,2} \mathbf{p}_{4,5}=-\mathbf{Z}_{4,5,2} \mathbf{Z}_{4,8,5} \mathbf{p}_{8,4}=-\boldsymbol{\Omega}_{4,8,5,2} \mathbf{p}_{8,4} \\
& \mathbf{p}_{4,6}=\mathbf{Z}_{4,7,6} \mathbf{p}_{4,7}=-\mathbf{Z}_{4,7,6} \mathbf{Z}_{4,8,7} \mathbf{p}_{8,4}=-\boldsymbol{\Omega}_{4,8,7,6} \mathbf{p}_{8,4} \\
& \mathbf{p}_{8,9}=\mathbf{Z}_{8,7,9} \mathbf{p}_{8,7}=\mathbf{Z}_{8,7,9} \mathbf{Z}_{8,4,7} \mathbf{p}_{8,4}=\boldsymbol{\Omega}_{8,4,7,9} \mathbf{p}_{8,4} \\
& \mathbf{p}_{2,1}=\mathbf{Z}_{2,3,1} \mathbf{p}_{2,3}=\mathbf{Z}_{2,3,1} \mathbf{Z}_{2,9,3} \mathbf{p}_{2,9}=\boldsymbol{\Omega}_{2,9,3,1} \mathbf{p}_{2,9}
\end{aligned}
$$

Since

$$
\mathbf{p}_{1,6}=-\mathbf{p}_{2,1}-\mathbf{p}_{4,2}+\mathbf{p}_{4,6}
$$

and

$$
\mathbf{p}_{2,9}=-\mathbf{p}_{4,2}-\mathbf{p}_{8,4}+\mathbf{p}_{8,9}
$$

then

$$
\begin{aligned}
\mathbf{p}_{1,6} & =-\boldsymbol{\Omega}_{2,9,3,1} \mathbf{p}_{2,9}+\boldsymbol{\Omega}_{4,8,5,2} \mathbf{p}_{8,4}-\boldsymbol{\Omega}_{4,8,7,6} \mathbf{p}_{8,4} \\
& =\left[-\boldsymbol{\Omega}_{2,9,3,1}\left(\boldsymbol{\Omega}_{4,8,5,2}+\boldsymbol{\Omega}_{8,4,7,9}-\mathbf{I}\right)+\boldsymbol{\Omega}_{4,8,5,2}-\boldsymbol{\Omega}_{4,8,7,6}\right] \mathbf{p}_{8,4} .
\end{aligned}
$$

Therefore,

$$
\operatorname{det}\left(-\boldsymbol{\Omega}_{2,9,3,1}\left(\boldsymbol{\Omega}_{4,8,5,2}+\boldsymbol{\Omega}_{8,4,7,9}-\mathbf{I}\right)+\boldsymbol{\Omega}_{4,8,5,2}-\boldsymbol{\Omega}_{4,8,7,6}\right)=\frac{s_{1,6}}{s_{4,8}} .
$$


The left hand side of the above equation is a function of the unknown squared distances $s_{2,9}$ and $s_{4,8}$. Since, from equation (30),

$$
s_{2,9}=\operatorname{det}\left(\boldsymbol{\Omega}_{4,8,5,2}+\boldsymbol{\Omega}_{8,4,7,9}-\mathbf{I}\right) s_{4,8},
$$

then the substitution of (32) in (31) yields a scalar equation in $s_{4,8}$ whose roots in the range in which the signed areas of the triangles $P_{4} P_{8} P_{5}$ and $P_{8} P_{4} P_{7}$ are real, that is, the range

$$
\left[\max \left\{\left(d_{5,8}-d_{4,5}\right)^{2},\left(d_{4,7}-d_{7,8}\right)^{2}\right\}, \min \left\{\left(d_{5,8}+d_{4,5}\right)^{2},\left(d_{4,7}+d_{7,8}\right)^{2}\right\}\right]
$$

determine the assembly modes of the analyzed linkage. These roots can be obtained, as in the previous section, for the four possible combinations of signs for the signed areas of the triangles $P_{4} P_{8} P_{5}$ and $P_{8} P_{4} P_{7}$. For each real root, we can determine the Cartesian position of the six revolute pair centers of the moving ternary links using equations (25)(28) and the equation $\mathbf{p}_{4,8}=\mathbf{Z}_{4,5,8} \mathbf{p}_{4,5}$. This process leads up to eight combinations of locations for $P_{1}$ and $P_{6}$, and at least one of them must satisfy the distance imposed by the binary link connecting them.

If a polynomial representation is still preferred, we can proceed as described next.

\subsection{Polynomial derivation}

By expanding all the Cayley-Menger determinants involved in equation (32), we get

$$
s_{2,9}=\frac{1}{s_{4,8}}\left(\Gamma_{1}+\Gamma_{2} A_{4,8,5}+\Gamma_{3} A_{8,4,7}+\Gamma_{4} A_{4,8,5} A_{8,4,7}\right)
$$

where

$$
\begin{aligned}
& A_{4,8,5}= \pm \frac{1}{2} \sqrt{\left[s_{4,8}-\left(d_{5,8}-d_{4,5}\right)^{2}\right]\left[\left(d_{5,8}+d_{4,5}\right)^{2}-s_{4,8}\right]} \\
& A_{8,4,7}= \pm \frac{1}{2} \sqrt{\left[s_{4,8}-\left(d_{4,7}-d_{7,8}\right)^{2}\right]\left[\left(d_{4,7}+d_{7,8}\right)^{2}-s_{4,8}\right]}
\end{aligned}
$$

and $\Gamma_{i}, i=1, \ldots, 4$, are polynomials in $s_{4,8}$ whose coefficients are algebraic functions of the known squared distances $s_{2,4}, s_{2,5}, s_{4,5}, s_{4,7}, s_{5,8}, s_{7,8}, s_{7,9}$, and $s_{8,9}$. On the other hand, by expanding equation (31), we obtain

$$
\begin{aligned}
& \Upsilon_{1}+\Upsilon_{2} A_{2,9,3}+\Upsilon_{3} A_{4,8,5}+\Upsilon_{4} A_{8,4,7}+\Upsilon_{5} A_{2,9,3} A_{4,8,5} \\
& +\Upsilon_{6} A_{2,9,3} A_{8,4,7}+\Upsilon_{7} A_{4,8,5} A_{8,4,7}+\Upsilon_{8} A_{2,9,3} A_{4,8,5} A_{8,4,7}-s_{1,6} s_{2,9} s_{4,8}=0
\end{aligned}
$$

where

$$
A_{2,9,3}= \pm \frac{1}{2} \sqrt{\left[s_{2,9}-\left(d_{3,9}-d_{2,3}\right)^{2}\right]\left[\left(d_{3,9}+d_{2,3}\right)^{2}-s_{2,9}\right]}
$$

and $\Upsilon_{i}, i=1, \ldots, 4$, are polynomials in the unknown distances $s_{2,9}$ and $s_{4,8}$ whose coefficients are algebraic functions of known squared distances. 
Now, by properly squaring equation (34), we obtain a polynomial equation in $s_{2,9}$ whose coefficients are radical expressions in $s_{4,8}$. Therefore, by replacing (33) in this polynomial equation, we get

$$
\frac{1}{s_{4,8}^{2}}\left(\Phi_{1}+\Phi_{2} A_{4,8,5}+\Phi_{3} A_{8,4,7}+\Phi_{4} A_{4,8,5} A_{8,4,7}\right)=0
$$

where $\Phi_{1}, \Phi_{2}, \Phi_{3}$, and $\Phi_{4}$ are polynomials in $s_{4,8}$ of degree $8,7,7$, and 6 , respectively. Finally, to obtain a polynomial equation, the square roots in (35) can be eliminated by properly twice squaring it. When the result is fully expanded, we obtain

$$
s_{4,8}^{2} s_{2,9} \Delta_{I I}=0,
$$

where $\Delta_{I I}$ is a 16 th-degree polynomial in $s_{4,8}$. The extraneous roots at $s_{2,9}=0$ and $s_{4,8}=0$ were introduced when clearing denominators to obtain equation (34), so they can be dropped. The degree of polynomial $\Delta_{I I}$ concurs with the result presented by $\mathrm{C}$. Innocenti in [7].

\subsection{Example}

According to the notation used in Fig. 6. let us suppose that $s_{1,2}=25, s_{1,3}=100$, $s_{1,6}=97, s_{2,3}=45, s_{2,4}=13, s_{2,5}=36, s_{3,9}=97, s_{4,5}=25, s_{4,6}=13, s_{4,7}=20$, $s_{5,8}=16, s_{6,7}=17, s_{7,8}=13, s_{7,9}=37$, and $s_{8,9}=20$. Substituting these values in $\Delta_{I I}$, the following characteristic polynomial is obtained

$$
\begin{aligned}
& 18.882510^{24} s_{4,8}^{16}-5.973510^{27} s_{4,8}^{15}+818.572210^{27} s_{4,8}^{14}-64.183710^{30} s_{4,8}^{13} \\
& +3.213710^{33} s_{4,8}^{12}-108.728510^{33} s_{4,8}^{11}+2.553110^{36} s_{4,8}^{10}-41.523910^{36} s_{4,8}^{9} \\
& +452.682410^{36} s_{4,8}^{8}-3.119610^{39} s_{4,8}^{7}+12.615410^{39} s_{4,8}^{6}-28.293610^{39} s_{4,8}^{5} \\
& +38.935310^{39} s_{4,8}^{4}-36.134110^{39} s_{4,8}^{3}+25.500710^{39} s_{4,8}^{2}-15.115110^{39} s_{4,8} \\
& +5.285410^{39} .
\end{aligned}
$$

The real roots of this polynomial are 1.1161, 1.2002, 7.3517, 10.4180, 17.0000, 27.5995, $52.9281,53.7863,56.0905$ and 61.5796 . The corresponding configurations for the case in which $\mathbf{p}_{2}=(0,0)^{T}, \mathbf{p}_{4}=(2,3)^{T}$, and $\mathbf{p}_{5}=(6,0)^{T}$ appear in Fig. 7 .

\section{Position analysis of the type III seven-link AKC}

Fig. 8 shows the general seven-link AKC of type III. If the quaternary link is assumed to be connected to the ground, the centers of its revolute pairs define the base quadrilateral $P_{1} P_{2} P_{6} P_{7}$, the revolute pair centers of the other two ternary links define the moving triangles $P_{3} P_{5} P_{4}$, and $P_{8} P_{5} P_{9}$. The position analysis problem for this linkage consists in, given the dimensions of every link and the position of the centers $P_{1}, P_{2}, P_{6}$, and $P_{7}$, calculating the Cartesian pose of the moving ternary links. 

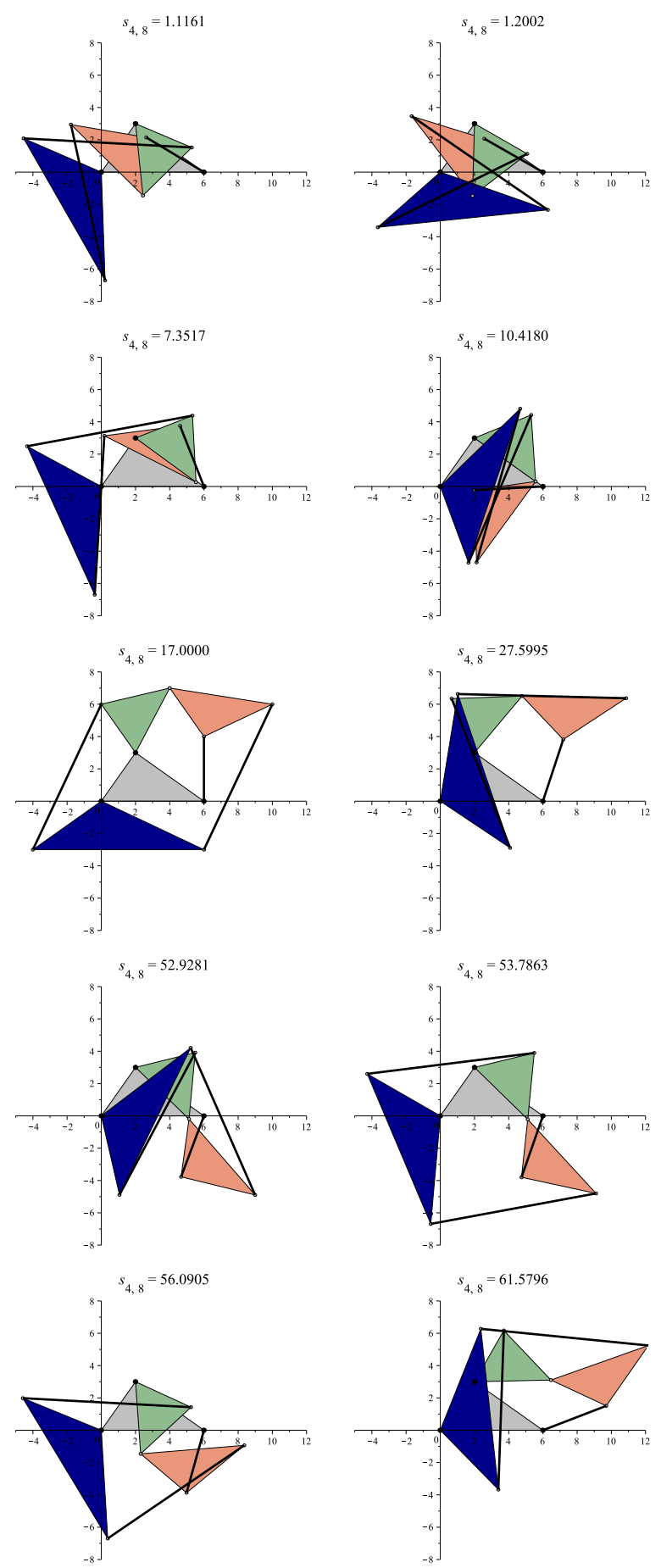

Figure 7: The assembly modes of the analyzed type II seven-link AKC. 


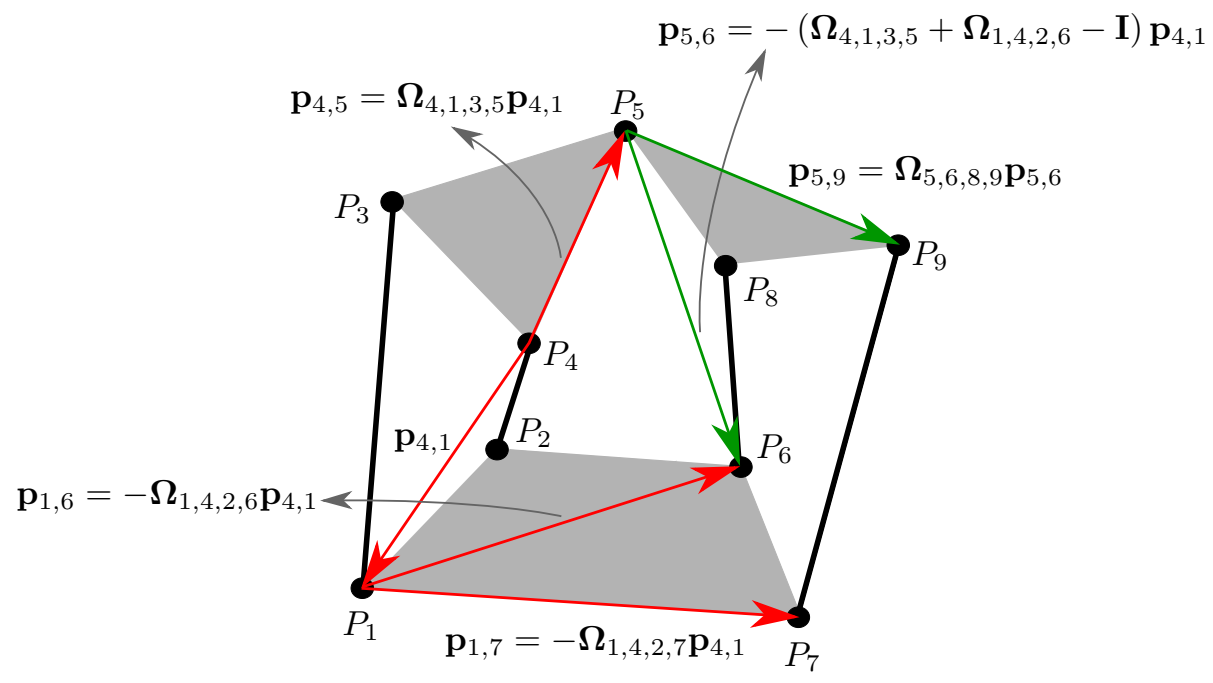

Figure 8: The seven-link AKC of type III. $\mathbf{p}_{7,9}$ can be expressed in function of $\mathbf{p}_{1,4}$ by computing eight bilaterations.

\subsection{Scalar equation derivation}

According to Fig. 8 , we have

$$
\begin{aligned}
& \mathbf{p}_{1,6}=\mathbf{Z}_{1,2,6} \mathbf{p}_{1,2}=-\mathbf{Z}_{1,2,6} \mathbf{Z}_{1,4,2} \mathbf{p}_{4,1}=-\boldsymbol{\Omega}_{1,4,2,6} \mathbf{p}_{4,1}, \\
& \mathbf{p}_{1,7}=\mathbf{Z}_{1,2,7} \mathbf{p}_{1,2}=-\mathbf{Z}_{1,2,7} \mathbf{Z}_{1,4,2} \mathbf{p}_{4,1}=-\boldsymbol{\Omega}_{1,4,2,7} \mathbf{p}_{4,1}, \\
& \mathbf{p}_{4,5}=\mathbf{Z}_{4,3,5} \mathbf{p}_{4,3}=\mathbf{Z}_{4,3,5} \mathbf{Z}_{4,1,3} \mathbf{p}_{4,1}=\boldsymbol{\Omega}_{4,1,3,5} \mathbf{p}_{4,1}, \\
& \mathbf{p}_{5,8}=\mathbf{Z}_{5,6,8} \mathbf{p}_{5,6}=\mathbf{Z}_{5,8,9} \mathbf{Z}_{5,6,8} \mathbf{p}_{5,6}=\boldsymbol{\Omega}_{5,6,8,9} \mathbf{p}_{5,6}
\end{aligned}
$$

Since

$$
\mathbf{p}_{7,9}=\mathbf{p}_{1,6}-\mathbf{p}_{1,7}-\mathbf{p}_{5,6}+\mathbf{p}_{5,9}
$$

and

$$
\mathbf{p}_{5,6}=-\mathbf{p}_{4,5}+\mathbf{p}_{4,1}+\mathbf{p}_{1,6},
$$

then

$$
\begin{aligned}
\mathbf{p}_{7,9} & =-\boldsymbol{\Omega}_{1,4,2,6} \mathbf{p}_{4,1}+\boldsymbol{\Omega}_{1,4,2,7} \mathbf{p}_{4,1}-\mathbf{p}_{5,6}+\boldsymbol{\Omega}_{5,6,8,9} \mathbf{p}_{5,6} \\
& =\left[-\boldsymbol{\Omega}_{1,4,2,6}+\boldsymbol{\Omega}_{1,4,2,7}-\left(\boldsymbol{\Omega}_{5,6,8,9}-\mathbf{I}\right)\left(\boldsymbol{\Omega}_{4,1,3,5}+\boldsymbol{\Omega}_{1,4,2,6}-\mathbf{I}\right)\right] \mathbf{p}_{4,1} .
\end{aligned}
$$

Therefore,

$$
\operatorname{det}\left(-\boldsymbol{\Omega}_{1,4,2,6}+\boldsymbol{\Omega}_{1,4,2,7}-\left(\boldsymbol{\Omega}_{5,6,8,9}-\mathbf{I}\right)\left(\boldsymbol{\Omega}_{4,1,3,5}+\boldsymbol{\Omega}_{1,4,2,6}-\mathbf{I}\right)\right)=\frac{s_{7,9}}{s_{1,4}} .
$$

The left hand side of the above equation is a function of the unknown squared distances $s_{1,4}$ and $s_{5,6}$. Since, from equation (42),

$$
s_{5,6}=\operatorname{det}\left(\boldsymbol{\Omega}_{4,1,3,5}+\boldsymbol{\Omega}_{1,4,2,6}-\mathbf{I}\right) s_{1,4},
$$


then the substitution of (44) in (43) yields a scalar equation in $s_{1,4}$ whose roots in the range in which the signed areas of the triangles $P_{1} P_{4} P_{2}$ and $P_{4} P_{1} P_{3}$ are real, that is, the range

$$
\left[\max \left\{\left(d_{2,4}-d_{1,2}\right)^{2},\left(d_{1,3}-d_{3,4}\right)^{2}\right\}, \min \left\{\left(d_{2,4}+d_{1,2}\right)^{2},\left(d_{1,3}+d_{3,4}\right)^{2}\right\}\right]
$$

determine the assembly modes of the analyzed linkage. As mentioned in previous sections, these roots can be obtained for the four possible combinations of signs for the signed areas of the triangles $P_{1} P_{4} P_{2}$ and $P_{4} P_{1} P_{3}$ but, if a polynomial representation is preferred, we can proceed as described next.

\subsection{Polynomial derivation}

Following the procedure described in the previous section for the polynomial derivation, from equations (43) and (44), we obtain

$$
\frac{1}{s_{1,4}^{2}}\left(\Phi_{1}+\Phi_{2} A_{1,4,2}+\Phi_{3} A_{4,1,3}+\Phi_{4} A_{1,4,2} A_{4,1,3}\right)=0
$$

where

$$
\begin{aligned}
& A_{1,4,2}= \pm \frac{1}{2} \sqrt{\left[s_{1,4}-\left(d_{2,4}-d_{1,2}\right)^{2}\right]\left[\left(d_{2,4}+d_{1,2}\right)^{2}-s_{1,4}\right]}, \\
& A_{4,1,3}= \pm \frac{1}{2} \sqrt{\left[s_{1,4}-\left(d_{1,3}-d_{3,4}\right)^{2}\right]\left[\left(d_{1,3}+d_{3,4}\right)^{2}-s_{1,4}\right]},
\end{aligned}
$$

and $\Phi_{1}, \Phi_{2}, \Phi_{3}$, and $\Phi_{4}$ are polynomials in $s_{1,4}$ of degree $8,7,7$, and 6 , respectively. Finally, by properly twice squaring the above equation, we get

$$
s_{1,4}^{2} s_{5,6} \Delta_{I I I}=0
$$

where $\Delta_{I I I}$ is a 18th-degree polynomial in $s_{1,4}$. The extraneous roots at $s_{1,4}=0$ and $s_{5,6}=0$ were introduced when clearing denominators in this polynomial derivation, so they can be dropped. The degree of polynomial $\Delta_{I I I}$ concurs with the result presented by C. Innocenti in [8].

Each of real roots of $\Delta_{I I I}$ determine the Cartesian position of the five revolute pair centers of the moving ternary links using equations (37)-(40) and the equation $\mathbf{p}_{1,4}=$ $\mathbf{Z}_{1,2,4} \mathbf{p}_{1,2}$. This process leads to up eight combinations of locations for $P_{7}$ and $P_{9}$, and at least one of them must satisfy the distance imposed by the binary link connecting them.

\subsection{Example}

According to the notation used in Fig. 8 let us suppose that $s_{1,2}=20, s_{1,3}=40$, $s_{1,6}=65, s_{1,7}=144, s_{2,4}=13, s_{2,6}=17, s_{2,7}=68, s_{3,4}=17, s_{3,5}=40, s_{4,5}=13$, $s_{5,8}=18, s_{5,9}=29, s_{6,7}=17, s_{6,8}=25, s_{7,9}=37$, and $s_{8,9}=5$. Substituting these values in $\Delta_{I I I}$, the following characteristic polynomial is obtained 

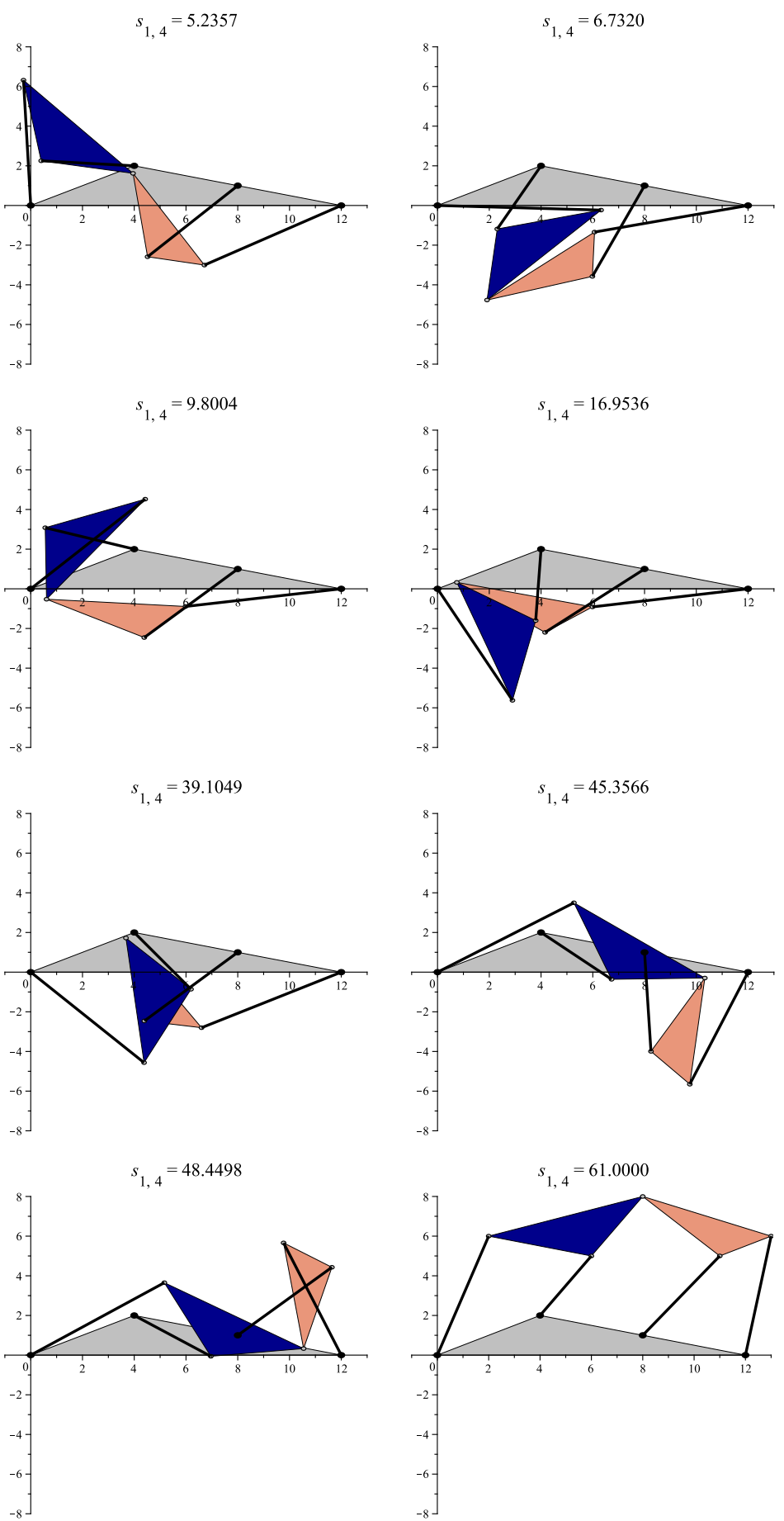

Figure 9: The assembly modes of the analyzed type III seven-link AKC. 


$$
\begin{aligned}
& -702.066910^{12} s_{1,4}^{18}+440.955110^{15} s_{1,4}^{17}-126.526010^{18} s_{1,4}^{16}+21.930610^{21} s_{1,4}^{15} \\
& -2.559210^{24} s_{1,4}^{14}+212.283510^{24} s_{1,4}^{13}-12.894510^{27} s_{1,4}^{12}+583.504410^{27} s_{1,4}^{11} \\
& -19.901010^{30} s_{1,4}^{10}+517.833110^{30} s_{1,4}^{9}-10.472510^{33} s_{1,4}^{8}+168.734010^{33} s_{1,4}^{7} \\
& -2.196110^{36} s_{1,4}^{6}+22.542010^{36} s_{1,4}^{5}-171.471710^{36} s_{1,4}^{4}+898.741510^{36} s_{1,4}^{3} \\
& -3.027910^{39} s_{1,4}^{2}+5.994210^{39} s_{1,4}-5.521810^{39} .
\end{aligned}
$$

The real roots of this polynomial are $5.2357,6.7320,9.8004,16.9536,39.1049,45.3566$, 48.4498, and 61.0000. The corresponding configurations for the case in which $\mathbf{p}_{1}=$ $(0,0)^{T}, \mathbf{p}_{2}=(4,2)^{T}, \mathbf{p}_{6}=(8,1)^{T}$, and $\mathbf{p}_{7}=(12,0)^{T}$ appear in Fig. 9]

\section{Conclusion}

The three seven-link Assur kinematic chains contain three independent kinematic loops. The standard approach for the position analysis of these chains consists in deriving the closure conditions for these three loops and obtaining an algebraic resultant. Nevertheless, formulating the position analysis in terms of kinematic loop equations introduces a major disadvantage: the resulting equations involve translations and rotations simultaneously. We have presented a different approach in which, instead of dealing with the Cartesian poses of the involved links, the position analysis problem is fully posed in terms of distances. Then, under this approach, the closure conditions boil down to a single distance ratio computable by bilaterations. An important simplification is thus obtained.

How the proposed approach scales to more complex Assur kinematic chains is an open problem. It is well-known that there are 28 AKCs with four loops or nine links [19]. The position analysis of some of these AKCs, based on either resultant elimination [20, 21, 22, 23] or homotopy continuation methods [9, 24], has already been reported in the literature. It can be checked that the required distances ratios for most of these chains can also be obtained by considering trees of triangles sharing only two vertices. Thus, the application of the proposed technique to the position analysis of the AKCs with nine links seems advantageous but this is certainly a point that deserves further attention.

\section{Acknowledgments}

We gratefully acknowledge the financial support of the Autonomous Government of Catalonia through the VALTEC program, cofinanced with FEDER funds, and the Colombian Ministry of Communications and Colfuturo through the ICT National Plan of Colombia.

\section{References}

[1] E. Peisach, Determination of the position of the member of threejoint and two-joint four member assur groups with rotational pairs (in russian), Machinowedenie (5) (1985) 55-61. 
[2] G. Pennock, D. Kassner, Kinematic analysis of a planar eight-bar linkage: Application to a platformtype robot, Journal of Mechanical Design 114 (1) (1992) 87-95.

[3] K. Wohlhart, Direct kinematic solution of a general planar stewart platform, in: Proceedings of the International Conference on Computer Integrated Manufacturing, Zakopane, Poland, 1992, pp. 403-411.

[4] C. Gosselin, J. Sefrioui, M. Richard, Solutions polynomiales au problème de la cinématique des manipulateurs parallèles plans à trois degrés de liberté, Mechanism and Machine Theory 27 (2) (1992) 107-119.

[5] N. Rojas, F. Thomas, A robust forward kinematics analysis of 3-rpr planar platforms, 12th international symposium on advances in robot kinematics, in: Advances in Robot Kinematics, 2010, pp. $23-32$.

[6] C. Innocenti, Position analysis in analytical form of the 7-link assur kinematic chain featuring one ternary link connected to ternary links only, Mechanism and Machine Theory 32 (4) (1997) $501-$ 509.

[7] C. Innocenti, Analytical-form position analysis of the 7-link assur kinematic chain with four seriallyconnected ternary links, Journal of Mechanical Design 116 (2) (1994) 622-628.

[8] C. Innocenti, Polynomial solution to the position analysis of the 7-link assur kinematic chain with one quaternary link, Mechanism and Machine Theory 30 (8) (1995) 1295 - 1303.

[9] A. Liu, T. Yang, Displacement analysis of planar complex mechanism using continuation method, Mechanical Science and Technology 13 (2) (1994) $55-62$.

[10] A. Dhingra, A. Almadi, D. Kohli, Closed-form displacement and coupler curve analysis of planar multi-loop mechanisms using gröbner bases, Mechanism and Machine Theory 36 (2) (2001) 273 298.

[11] J. Nielsen, B. Roth, Solving the input/output problem for planar mechanisms, Journal of Mechanical Design 121 (2) (1999) 206-211.

[12] C. Wampler, Solving the kinematics of planar mechanisms by dixon determinant and a complexplane formulation, Journal of Mechanical Design 123 (3) (2001) 382-387.

[13] J. Verschelde, Algorithm 795: PHCPACK: A general-purpose solver for polynomial systems by homotopy continuation, ACM Transactions on Mathematical Software 25 (2) (1999) 251-276.

[14] D. Bates, J. Hauenstein, A. Sommese, C. Wampler, Bertini: Software for numerical algebraic geometry. available at http://www.nd.edu/ sommese/bertini/.

[15] A. Sommese, C. Wampler, The Numerical Solution of Systems of Polynomials Arising in Engineering and Science, World Scientific, 2005.

[16] J. Merlet, Alias: An interval analysis based library for solving and analyzing system of equations, in: Proceedings of the SEA, Toulouse, France, 2000.

[17] J. Porta, L. Ros, F. Thomas, A linear relaxation technique for the position analysis of multiloop linkages, IEEE Transactions on Robotics 25 (2) (2009) $225-239$

[18] F. Thomas, L. Ros, Revisiting trilateration for robot localization, IEEE Transactions on Robotics 21 (1) (2005) 93-101.

[19] T. Yang, F. Yao, Topological characteristics and automatic generation of structure analysis and synthesis of plane mechanisms, part i -theory, part ii -application, in: Proceedings of the ASME Mechanisms Conference, Vol. 15, Kissimmee, FL, 1988, pp. $178-190$

[20] L. Han, Q. Liao, C. Liang, A kind of algebraic solution for the position analysis of a planar basic kinematic chain, Journal of Machine Design 16 (3) (1999) 16 - 18.

[21] L. Han, Q. Liao, C. Liang, Closed-form displacement analysis for a nine-link barranov truss or a eight-link assur group, Mechanism and Machine Theory 35 (3) (2000) 379 - 390.

[22] P. Wang, Q. Liao, Y. Zhuang, S. Wei, A method for position analysis of a kind of nine-link barranov truss, Mechanism and Machine Theory 42 (10) (2007) $1280-1288$.

[23] P. Wang, Q. Liao, Y. Zhuang, S. Wei, Research on position analysis of a kind of nine-link barranov truss, Journal of Mechanical Design 130 (1) (2008) 011005.

[24] L. Hang, Q. Jin, T. Yang, A general study of the number of assembly configurations for multi-circuit planar linkages, Journal of Southeast University 16 (1) (2000) $46-51$ 